\title{
Position Location Estimation Algorithms for Mobile Station in NLOS Propagation Environment
}

\author{
Xiaohua Tian, Juanjuan Yin \\ School of intelligence Science and Information Engineering \\ Xi'an Peihua University, Xi'an, China \\ 1484472221@ qq.com,294539515@qq.com
}

Key words: Time of arrival (TOA); Angle of arrival (AOA); Position location (PL); Line of sight (LOS) propagation; Non-line-of-sight (NLOS) propagation; Feasible region.

\begin{abstract}
The non-line-of-sight (NLOS) propagation is a key problem of subscriber location in cellular networks. The angle of arrival (AOA) and time/angle of arrival (TOA/AOA) position location (PL) estimation methods are presented to mitigate the NLOS propagation in this paper. In order to reduce the feasible region of the mobile station (MS) and to improve the accuracy of the location estimation, the angle spread measured AOA is constrained by the geometrically based single bounce circular model (GBSBCM) and the measured TOA is constrained by the fact that the measured TOA is always greater than the true TOA value. Its effectiveness is verified by the computer simulation.
\end{abstract}

\section{Introduction}

Wireless position location has received considerable attention over the past few years due to the united states federal communications commission's(FCC) mandate requires that all wireless service providers, including cellular, personal communications services(PCS), and some special mobile radio(SMR) licensees, provide location information for Enhanced-911(E-911) safety services [1,2]. Location service has been a basic requirement in mobile communication system. Because the NLOS propagation is the main cause of positioning error in cellular networks, mitigating the influence of the NLOS propagation and improving the location accuracy are researched [3-6, 11-23]. These techniques are mainly based on TOA PL system.

It is possible that using measured AOA parameters estimates position location of the MS in cellular networks used smart antennas in the base station (BS), and AOA PL method not need precise reference time relative to TOA PL method. Because the NLOS can introduce large errors in the AOA-based location estimates, it is necessary to study on AOA PL estimation algorithms of the MS for the NLOS propagation environment. AOA and TOA/AOA PL estimation methods are presented to mitigate the NLOS propagation in this paper.

\section{Geometrically Based Single Bounce Statistical Channel Models}

Geometrically based single bounce (GBSB) statistical channel Models are developed by defining a spatial scatterer density function [7]. These models are useful for both simulation and analysis purpose. There is the geometrically based single bounce circular model (GBSBCM) in these models. Besides cellular networks design and performance analysis, the model is useful for AOA PL estimation algorithms analysis of cellular networks used smart antennas in the BS. The geometry of the GBSBCM is shown in Figure 1. The model is applicable to high tier, macro-cell scenarios where the BS is very high relative to scatterers, and is based on a circular distribution of scatterers near the MS. It assumes that the scatterers lie within radius $\mathrm{Rm}$ about the mobile. Often the requirement that $\mathrm{Rm}<\mathrm{D}$ is imposed. The model is based on the assumption that in macro-cell environments where antenna heights are relatively high, there will be no signal scattering from locations near the BS. The maximum delay $\tau$ max and the maximum angle spread $\theta$ max are as shown in the following formula. 


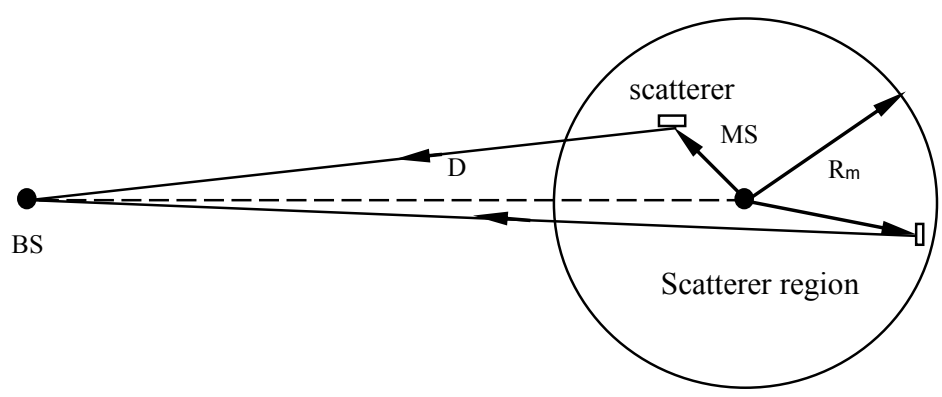

Figure 1 The geometrically based single bounce circular model

$$
\begin{aligned}
\tau_{\text {max }} & =2 R_{m} / c \\
\theta_{\max } & =\arcsin \left(\frac{R_{m}}{D}\right)=\arcsin \left(\frac{R_{m}}{\sqrt{\left(x-x_{i}\right)^{2}+\left(y-y_{i}\right)^{2}}}\right)
\end{aligned}
$$

where $\mathrm{c}$ is the speed of light.

Apparently, the parameter $\tau$ max is determined by scatterers radius $\mathrm{Rm}$, and the parameter $\theta$ max is determined by scatterers radius $\mathrm{Rm}$ and T-R range. When positions of $\mathrm{BS}$ and MS are given, $\theta \max$ is determined by scatterers radius $\mathrm{Rm}$. Scatterers radius $\mathrm{Rm}$ can be obtained by measurement or COST-207 model [8]. The power delay characteristic of the COST-207 model for receiving broad band signal in the urban macro-cell environment is shown in Table 1 . The maximum delay is 5us and number of multipath signals is 6 in the table.

Table 1. COST-207 6-tap reduced typical urban power delay profile.
\begin{tabular}{|c|c|c|c|c|c|c|}
\hline Delay(us) & 0.0 & 0.2 & 0.5 & 1.6 & 2.3 & 5.0 \\
\hline $\begin{array}{c}\text { Fractional } \\
\text { Power }\end{array}$ & 0.189 & 0.379 & 0.239 & 0.095 & 0.061 & 0.037 \\
\hline
\end{tabular}

\section{PL Estimation Algorithms for NLOS Propagation in Cellular Networks}

\subsection{The Time-Based Location Algorithm}

Signals received by the BS in the NLOS propagation environment consist of multipath components which have different TOA and AOA parameters. The TOA PL estimation algorithm is presented to mitigate the NLOS effect in literature [5], in which the facts that the range error is always positive and TOA of the first multipath signal is always most close to that of LOS propagation are used. The algorithm estimates the location of the MS using all TOAs of the first multipath signal and improves the accuracy of the location estimation by the inequality restrictions to reduce the feasible region of the MS. Since AOA of the first multipath signal is not always most close to that of LOS propagation among all multipath signals in NLOS propagation environment, in order to improve the positioning accuracy, the AOA-based location algorithm is presented in this paper, using AOAs of all multipath signals to mitigate the NLOS effect by the inequality restrictions in (2) to reduce the feasible region of the MS. The feasible region (shaded region) of the MS is shown in Fig. 2.

The AOA-based location algorithm assumes that the MS, located at (x,y), transmits its sequence The $\mathrm{N}$ base stations receivers located at coordinates $\left(\mathrm{x}_{1}, \mathrm{y}_{1}\right),\left(\mathrm{x}_{2}, \mathrm{y}_{2}\right), \ldots,\left(\mathrm{x}_{\mathrm{N}}, \mathrm{y}_{\mathrm{N}}\right)$ receive the sequence. Let $\theta_{\mathrm{ij}}$ be the AOA of the jth multipath signal measured by the ith BS, and $\varepsilon_{\mathrm{ij}}$ be its corresponding error. The relation between $\theta_{\mathrm{ij}}$ and $\varepsilon_{\mathrm{ij}}$ is as follows.

$$
\theta_{i j}=\operatorname{arctg}\left(\frac{y_{i}-y}{x_{i}-x}\right)+\varepsilon_{i j} \quad \mathrm{i}=1,2, \ldots, \mathrm{N}, \mathrm{j}=1,2,3
$$

Considering the fact that all angle spreads are less than the maximum angle spread, mathematically, there is the following inequality. 


$$
\left|\theta_{i j}-\operatorname{arctg}\left(\frac{y_{i}-y}{x_{i}-x}\right)\right| \leq \arcsin \left(\frac{R_{m}}{\sqrt{\left(x_{i}-x\right)^{2}+\left(y_{i}-y\right)^{2}}}\right) \quad ; \mathrm{i}=1,2, \ldots, \mathrm{N}, \mathrm{j}=1,2,3
$$

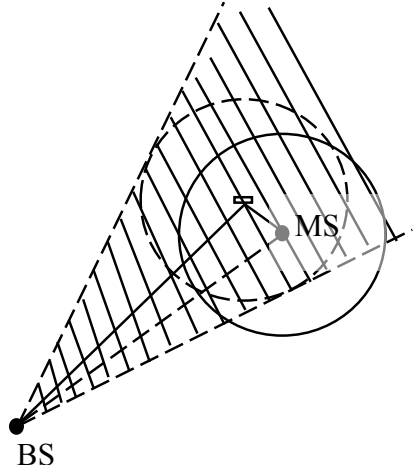

(a)

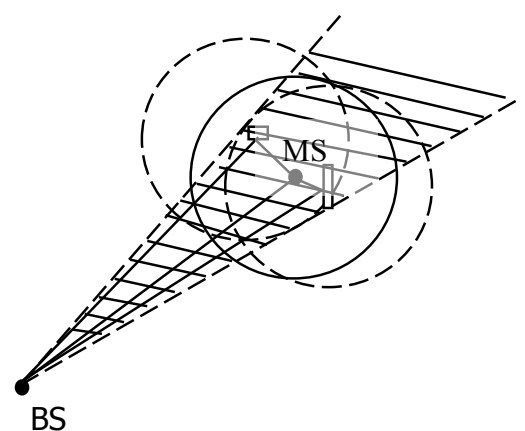

(b)

Figure 2 The feasible region(shaded region) of the MS ( The feasible region determined by only one AOA parameter in (a); The feasible region determined by two AOA parameters in (b))

The AOA-based PL estimation in the NLOS propagation environment is converted to nonlinear least squares (NL-LS) problems with nonlinear inequality constraints. Mathematically, there is the following function.

$$
\left\{\begin{array}{l}
F(x, y)=\min _{x, y} \sum_{i=1}^{N} \sum_{j=1}^{3}\left|\theta_{i j}-\operatorname{arctg}\left(\frac{y_{i}-y}{x_{i}-x}\right)\right|^{2} \\
\text { s.t. }\left|\theta_{i j}-\operatorname{arctg}\left(\frac{y_{i}-y}{x_{i}-x}\right)\right| \leq \arcsin \left(\frac{R_{m}}{\sqrt{\left(x_{i}-x\right)^{2}+\left(y_{i}-y\right)^{2}}}\right) \\
\mathrm{i}=1,2, \ldots, \mathrm{N}, \mathrm{j}=1,2,3
\end{array}\right.
$$

There are many approaches to forming numerical solutions for NL-LS problems with nonlinear inequality constraints. One simple, yet effective, method uses penalty functions to modify the objective function and form a solution using an unconstrained approach [9]. The penalty functions provide a large penalty to objective function when one or more of the constraints are violated. The objective function including the penalty function is as follows.

$$
P(x, y)=F(x, y)+\lambda \sum_{i=1}^{N} \sum_{j=1}^{3} \frac{1}{g_{i, j}(x, y)}
$$

where

$$
g_{i, j}(x, y)=\arcsin \left(\frac{R_{m}}{\sqrt{\left(x_{i}-x\right)^{2}+\left(y_{i}-y\right)^{2}}}\right)-\left|\theta_{i j}-\operatorname{arctg}\left(\frac{y_{i}-y}{x_{i}-x}\right)\right|,
$$

$\lambda$ is positive for minimization. As any constraint is approached during the search, the penalty term forces toward infinity, thus forming a natural optimum within the feasible region. This approach requires that the initial guess be placed within the feasible region. The initial guess can be determined by the solution of (3) in this paper, and the solution of (3) can be obtained by iterative search in literature [10].

\subsection{The TOA/AOA PL Estimation Algorithm}

Considering the facts that TOA of the first multipath signal is always most close to that of LOS propagation and AOA of the first multipath signal is not always most close to that of LOS propagation among all multipath signals in NLOS propagation environment, the TOA/AOA PL estimation algorithm presented in this paper estimates the location of the MS using TOAs of the first multipath signals measured by all BS and all AOAs measured by the host BS. The algorithm improves the accuracy of the location estimation by the inequality restrictions to reduce the feasible region of the MS. Constraint inequality of AOA parameter is formula (2) and constraint of TOA parameter is inequality which the range error is always positive. 
The algorithm assumes that the MS, located at (x, y), transmits its sequence at time $\tau_{0}$. The $\mathrm{N}$ base stations receivers located at coordinates $\left(\mathrm{x}_{1}, \mathrm{y}_{1}\right),\left(\mathrm{x}_{2}, \mathrm{y}_{2}\right), \ldots,\left(\mathrm{x}_{\mathrm{N}}, \mathrm{y}_{\mathrm{N}}\right)$ receive the sequence. Let $\tau_{i}$ be the TOA of the first multipath signal measured by the ith BS, and $\theta_{j}$ be the AOA of the jth multipath signal measured by host BS. Errors of measuring are as follows.

$$
\begin{aligned}
& f_{j}(x, y)=\theta_{j}-\operatorname{arctg}\left(\frac{y-y_{1}}{x-x_{1}}\right) \quad \mathrm{j}=1,2,3 \\
& g_{i}\left(x, y, \tau_{0}\right)=c\left(\tau_{i}-\tau_{0}\right)-\sqrt{\left(x-x_{i}\right)^{2}+\left(y-y_{i}\right)^{2}} \quad \mathrm{i}=1,2, \ldots, \mathrm{N}
\end{aligned}
$$

Considering the fact that angle spreads of multipath signals are less than the maximum angle spread determined by GBSBCM, mathematically, there are following inequalities.

$$
\left|\theta_{j}-\operatorname{arctg}\left(\frac{y-y_{1}}{x-x_{1}}\right)\right| \leq \arcsin \left(\frac{R_{m}}{\sqrt{\left(x-x_{1}\right)^{2}+\left(y-y_{1}\right)^{2}}}\right) \quad \mathrm{j}=1,2,3
$$

Since the range error is always positive and TOA of the first multipath signal is always most close to that of LOS propagation, there is the following inequality.

$$
g_{i}\left(x, y, \tau_{0}\right)=c\left(\tau_{i}-\tau_{0}\right)-\sqrt{\left(x-x_{i}\right)^{2}+\left(y-y_{i}\right)^{2}} \geq 0 \quad \mathrm{i}=1,2, \ldots, \mathrm{N}
$$

The TOA/AOA PL estimation in the NLOS propagation environment is converted to nonlinear least squares (NL-LS) problems with nonlinear inequality constraints. Mathematically, there are the following functions.

$$
\left\{\begin{array}{l}
F\left(x, y, \tau_{0}\right)=\min _{x, y, \tau_{0}}\left(\sum_{j=1}^{3}\left|\theta_{j}-\operatorname{arctg}\left(\frac{y-y_{1}}{x-x_{1}}\right)\right|^{2}+\sum_{i=1}^{N}\left|c\left(\tau_{i}-\tau_{0}\right)-\sqrt{\left(x-x_{i}\right)^{2}+\left(y-y_{i}\right)^{2}}\right|^{2}\right) \\
\text { s.t. } \quad\left|\theta_{j}-\operatorname{arctg}\left(\frac{y-y_{1}}{x-x_{1}}\right)\right| \leq \arcsin \left(\frac{R_{m}}{\sqrt{\left(x-x_{1}\right)^{2}+\left(y-y_{1}\right)^{2}}}\right) \\
\quad c\left(\tau_{i}-\tau_{0}\right)-\sqrt{\left(x-x_{i}\right)^{2}+\left(y-y_{i}\right)^{2}} \geq 0
\end{array}\right.
$$

$$
\mathrm{i}=1,2, \ldots, \mathrm{N}, \mathrm{j}=1,2,3
$$

Numerical solutions for above NL-LS problems with nonlinear inequality constraints can be obtained by using the same method as formula (5). The initial guess can be determined by the solution of (7) and (8), and the solution of (7) and (8) can be obtained by iterative search in literature [10].

\section{Simulation}

These algorithms effectiveness is verified by the computer simulation. We assume that radius of hexagonal macrocells is $5 \mathrm{~km}$ and coordinates of $5 \mathrm{BS}$ are $(0,0) \mathrm{m},(0,8660) \mathrm{m},(7500,4330) \mathrm{m}$, $(7500,-4330) \mathrm{m}$ and $(0,-8660) \mathrm{m}$. Position location of the MS is randomly generated. Number of multipath signals received by each BS is 3 and multipath signals are formed by scatterers which are uniformly distributed in near the MS. Angle errors are caused by the NLOS propagation only in simulation.

Simulation 1 verifies the performance of presented AOA-based PL method and analyzes the relation between average PL error and radius of scatterer region, number of the BS. Simulation results are obtained by 500 times Monte-Carlo experiments. The relation between average PL error and radius of scatterer region is shown in Fig. 3. It shows that average PL error will reduce when the number of the BS increases or radius of scatterer region reduces. Performance comparison presented the AOA-based PL method with conventional NL-LS method is shown in Fig. 4. It shows that presented method has less PL error than conventional method in the NLOS environment.

Simulation 2 verifies performance of presented AOA/TOA PL method and analyzes the relation between average PL error and radius of scatterer region, number of the BS. Simulation results are obtained by 500 times Monte-Carlo experiments and its results is shown in Fig. 5. For presented AOA/TOA PL method, the same conclusion is formed as presented AOA-based PL method. 


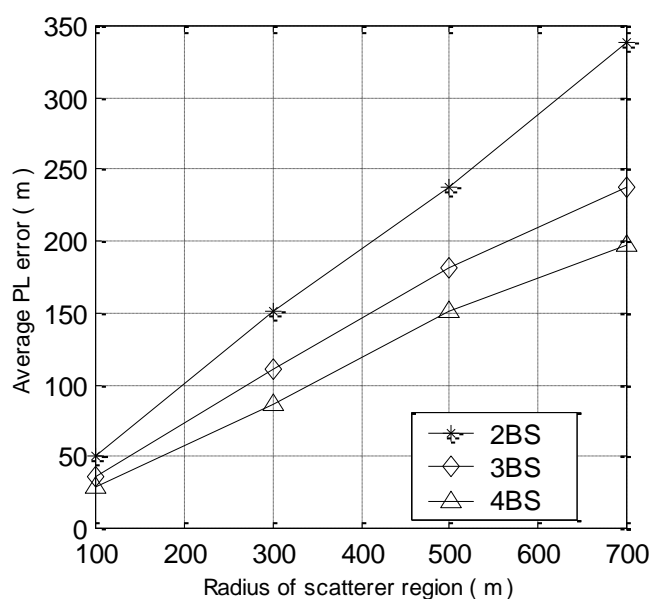

Figure 3 The relation between PL error of presented AOAbased method and radius of scatterer region, number of ths BS

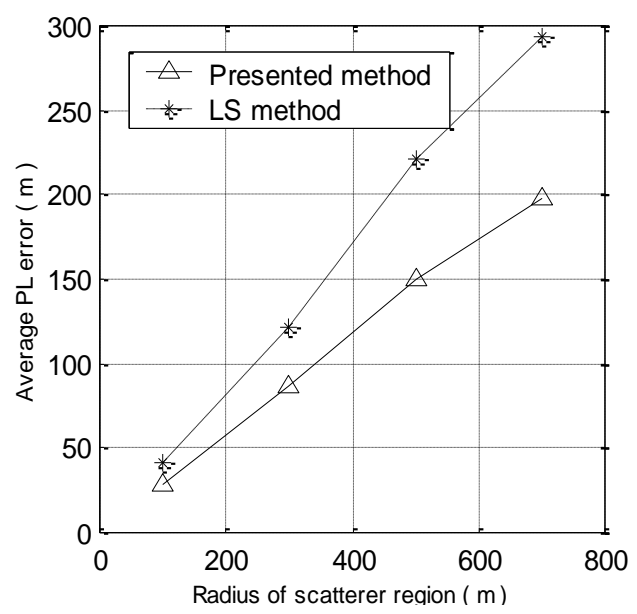

Figure 4 Performance comparison presented AOAbased method with conventional method (4 BS)

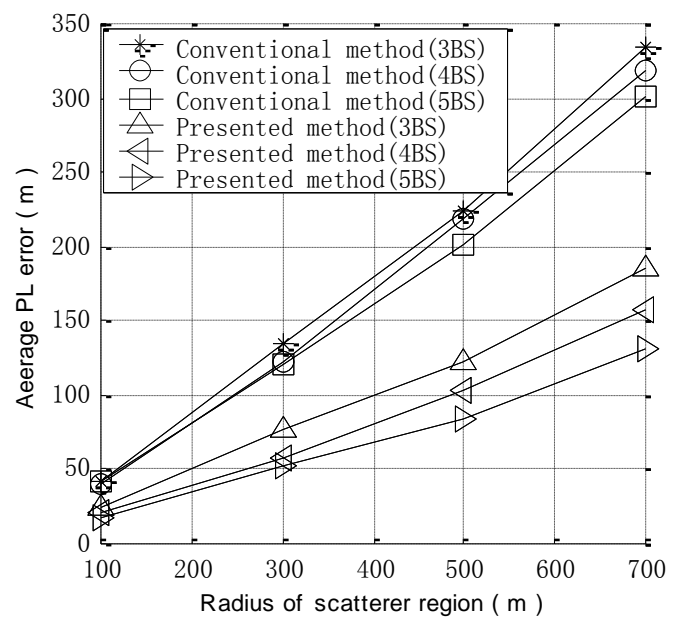

Figure 5 Performance comparison presented TOA/AOA method with conventional method. simulation.

\section{Acknowledgment}

This work was supported by the Education Department of Shaanxi Provincial Government under the Grant No.17Jk1054.

\section{References}

[1] T. S. Rappaport, J. H. Reed, and B. D. Woerner, Position location using wireless communications on highways of the future, IEEE Commun. Mag., 1996, pp. 33-41.

[2] J. Caffery, L. Stuber, Overview of radiolocation in CDMA cellular systems, IEEE Commun. Mag., 1998, pp. 38-45.

[3] M. Wylie and J. Holtzmann, The NLOS-Line of sight problem in mobile location estimation, in IEEE International Conference on Universal Personal Communications, 1996, pp.827-831.

[4] J. Caffery, Wireless location in CDMA cellular radio systems, Chap.7, Kluwer Academic Publishers, 2000.

[5] J. Caffery, L. Stuber, Subscriber location in CDMA cellular networks, IEEE Trans. on Vehicular Technology, Vol.47, No.2 ,1998, pp.406-415.

[6] X. H. Tian, G. S. Liao, An effective TOA-based location method for mitigating the influence of the NLOS propagation, Acta Electronica Sinica, Vol.31, No.9, 2003, pp.1429-1432. 
[7] R. Ertel, P. Cardieri, K. W. Sowerby, T. S. Rappaport, J. H. Reed, Overview of spatial channel models for antenna array communication systems, IEEE Personal Communications, Vol.5, No.1, Feb.1998, pp.10-22.

[8] J. Parsons, The mobile radio propagation channel, Halsted, 1992.

[9] Y. X. Yuan, W. Y. Sun, Optimization theory and method, Science Press, 2001, Chapter 10.

[10] W. H. Foy, Position-location solutions by Taylor-Series estimation, IEEE Trans. AES., vol. AES-12,Mar. 1976, pp. 187-193.

[11]Kenneth W. K. Lui et al. Maximum A Posteriors Approach to Time-of-Arrival-Based Localization in Non-Line-of-Sight Environment. IEEE Trans on VT., Vol. 59, No. 3, 2010.

[12]B T Sieskul et al. A Hybrid SS-ToA Wireless NLoS Geolocation Based on Path Attenuation: ToA Estimation and CRB for Mobile Position Estimation. IEEE Trans. on VT., Vol. 58, No. 9 , pp: 4930- 4942. 2009.

[13]Yaqin Xie et al. Grid-Search-Based Hybrid TOA/AOA Location Techniques for NLOS Environments. IEEE Communications Letters, Vol. 13, No. 4, 2009.

[14]Chien-Hua Chen et al. Wireless Location Estimation With the Assistance of Virtual Base Stations. IEEE Trans on VT., Vol. 58, No. 1, 2009.

[15]Ulrich Hammes et al. Robust Tracking and Geolocation for Wireless Networks in NLOS Environments IEEE Journal of Selected Topics in SP., Vol. 3, No. 5, 2009.

[16]Kai-Ten Feng et al. An Enhanced Geometry-Assisted Location Estimation Algorithm for NLOS Environments IEEE Trans on Mobil Computing, Vol. 7, No. 2, 2008.

[17]Kegen $\mathrm{Yu}$ et al. Improved Positioning Algorithms for Nonline-of- Sight Environments. IEEE Trans on VT., Vol. 57, No. 4, 2008.

[18]Changlin Ma et al. A Nonline-of-Sight Error-Mitigation Method for TOA Measurements. IEEE Ttrans. VT,Vol.56,No.2,pp:641-652, 2007.

[19]Wang Wei et al. A New NLOS Error Mitigation Algorithm in Location Estimation .IEEE Trans. on VT.Vol. 54, No. 6, pp:2048- 2053 , 2005.

[20]Saipradeep Venkatraman et al. A Novel ToA Location Algorithm Using LoS Range Estimation for NLoS Environments. IEEE Trans. VT,Vol.53, No.5,pp:1515-1524, 2004.

[21]Yiu-Tong Chan et al. Time-of-Arrival Based Localization Under NLOS Conditions IEEE Trans on VT Vol 55, No. 1,2006.

[22]Xin Wang et al. A TOA-Based Location Algorithm Reducing the Errors Due to Non-Line-ofSight (NLOS) Propagation. IEEE Trans.VT,Vol.52, No.1, pp:112-117,2003.

[23]Saleh Al-Jazzar et al. Scattering-Model-Based Methods for TOA Location in NLOS Environments IEEE Trans on VT. Vol 56, No. 2, 2007. 